\title{
Proceedings of the summer meeting of the Scottish Otolaryngological Society, Dunkeld, Scotland, UK, 9 June 2006
}

\author{
Teleconferencing in the management of head and neck \\ cancer: the west Scotland experience \\ I Nixon, S A Savage, K MacKenzie \\ From the Glasgow Royal Infirmary
}

The current guidelines produced by the British Association of Otorhinolaryngologists Head and Neck Surgeons in 2002 and the National Institute of Clinical Excellence in 2004 encourage the formation of cancer networks to provide management for head and neck cancer. Managed clinical networks have been introduced into Scotland to comply with these guidelines. Each network consists of multi-disciplinary teams which work together to provide a high standard of care for their patients.

The west of Scotland managed clinical network for head and neck cancer is the first network to use teleconferencing to facilitate a monthly cross-network clinical meeting. The meeting is used as a platform for clinicians to present challenging, interesting and educational cases.

We present an audit of the clinical meetings of the west of Scotland managed clinical network for head and neck cancer. These monthly meetings bring together seven multi-disciplinary teams from across the region in order to discuss a variety of cases and issues.

This presentation briefly describes the managed clinical network, the meetings and their format. We then go on to discuss the personnel involved in presenting at the meetings, the cases discussed, the issues raised and an audit of the meeting.

One hundred and twenty-two new patients have been discussed over 23 meetings. Their ages have ranged from 11 to 87 years, with a male:female ratio of 5:1. Pathology involving sites throughout the head and neck has been presented. Although the most common type of pathology is squamous cell carcinoma, a wide variety of other pathologies have also been discussed.

The issues discussed at the meetings have included: treatment advice, treatment validation, network policy, education and miscellaneous others. We go on to give examples of the cases and issues discussed.

An audit of the participants' views proved that the meetings were well received. The quality of presentation afforded by telecommunication was generally high, and network members found the meetings useful for patient management, discussion with other specialties and education.

These meetings have proved extremely successful, and we hope they have led to improvements in patient care. An ongoing database of new registrations will link with the clinical meetings and provide a valuable resource for future audit and research.

\section{Audit of compliance of the operative record with Royal} College of Surgeons of England guidelines

M R Humphreys, A S Evans, G Robertson*
From the Department of Otolaryngology, Head and Neck Surgery, Aberdeen Royal Infirmary, Aberdeen, and the ${ }^{*}$ Department of Otolaryngology, Head and Neck Surgery, Western General Hospital, Edinburgh, Scotland, UK

\section{Objectives}

We aimed to examine compliance with the Royal College of Surgeons of England guidelines for operative recordkeeping, before and after a period of education.

\section{Methods}

The study took the form of a completed audit cycle within the department of otolaryngology, head and neck surgery at the Western General Hospital, Edinburgh, Scotland.

The agreed outcome measure was an assessment of compliance with published guidelines for operative recordkeeping, compared before and after educational reminders.

We included 200 operative records for patients undergoing general ENT procedures between February and August 2005.

Results

There was a substantial improvement in the inclusion and legibility of six out of the eleven Royal College of Surgeons of England essential features of the operative record. Basic patient identification details recorded in the operative note also improved.

\section{Conclusions}

The use of educational presentations and reminder sheets are of value in improving compliance with Royal College of Surgeons of England guidelines on operative notekeeping. Despite education, some areas failed to improve.

\section{Sterimar vs xylometazoline in nasal surgical aftercare: a} blinded, randomized, comparative trial

A Evans, M Humphreys, C Y Eng, S McKean, D Grant From the Department of Otolaryngology, Aberdeen Royal Infirmary, Aberdeen, Scotland, UK

Patients undergoing nasal surgery commonly experience symptoms related to swelling and crusting in the postoperative period. A variety of topical preparations are used for symptomatic relief.

We conducted a blinded, randomized, comparative trial of the effects of xylometazoline $v s$ Sterimar ${ }^{\circledR}$ physiological sea water microspray on symptoms following septoplasty and functional endoscopic sinus surgery. One hundred and twenty patients were recruited from November 2003 to January 2006. Symptoms were recorded on a visual analogue scale at day 10.

Controlling for age, sex and operation type, no significant differences were found for nasal discharge $(p=0.71)$, sense of smell $(p=1.0)$, bleeding $(p=0.87)$ or blockage $(p=0.72)$. A significant difference was observed in median pain scores $(p=0.03)$, with less pain experienced in the Sterimar group. 
We did not find sufficient evidence to support the preferential use of one product over the other.

\section{Hand-washing in otolaryngology}

\section{G Robertson, I F Hathorn, A T Williams}

From the Department of Otolaryngology, Head and Neck Surgery, St John's Hospital, Howden, and The Royal Infirmary of Edinburgh, Edinburgh, Scotland, UK

\section{Introduction}

Each year, 33000 Scottish patients are affected by hospital-acquired infections. Hand-washing remains the most important measure in preventing the transmission of hospital-acquired infections. ${ }^{1}$ Despite this, hand-washing is still seldom performed. ${ }^{2}$ Otolaryngology out-patient consultations require frequent examination and instrumentation of the upper aerodigestive tract. This may represent a potential vector for the spread of upper respiratory tract pathogens in the community. While hospital-acquired infections and hospital hygiene have attracted a great deal of publicity in recent times, this is, to our knowledge, the first study of hand-washing practice in otolaryngology.

\section{Methods}

The study took place within the otolaryngology out-patient department at the Royal Infirmary of Edinburgh. One hundred consecutive out-patient consultations were analysed by an observer. The clinicians observed were of varying grades and were blinded to the analyses. The procedure and examination carried out were recorded, along with any observed hand-washing and use of gloves.

After the first analysis, an education programme was implemented and alcohol gel was made available. The study was then repeated.

\section{Results}

Prior to education, hand-washing was carried out in 43 per cent of consultations. Following education, this figure rose to 87 per cent.

Data were also obtained on hand-washing and glove use for individual procedures, and these will be presented.

\section{Discussion}

The incidence of hand-washing in this study was comparable with that in the literature. The improvement in the second study was mainly attributable to increased use of alcohol gel following a successful education programme.

Infection control is an important contemporary issue, and the National Health Service Quality Insurance Scotland organization advises that hospitals should test the effectiveness of measures put in place to encourage hand-washing and then publish their findings.

\section{References}

1 Reybrouck G. Role of the hands in the spread of nosocomial infections. J Hosp Infect 1983;4:103-10

2 Pittet D, Hugonnet S, Harbath S, Mourouga P, Sauvan V, Touveneau S et al. Effectiveness of a hospital-wide programme to improve compliance with hand hygiene. Lancet 2000;356:1307-12

\section{The sacculo-colic reflex test in otosclerosis and following stapes surgery}

E Stapleton

From the ENT Department, Ninewells Hospital, Dundee, Scotland, UK

The sacculo-colic test is said to assess otolith function. A myogenic response (in the ipsilateral sternocleidomastoid muscle) is measured when a sound stimulus is applied to the test ear, which is thought to arise as the result of a pressure wave in the fluid of the vestibule, produced by stapes displacement. If this is correct, one would not expect a response in cases of stapes fixation, and one might expect abnormal results in patients who have undergone stapedectomy.

Forty patients (44 stapedectomies, 33 otosclerotic ears, three normal ears) and 20 controls underwent repeated sacculo-colic tests.

The results confirmed the proposed mechanism of the sacculo-colic reflex test. The study also suggests that stapedectomy might cause saccular damage, or that the stapedectomy piston prosthesis is insufficient to generate a saccular pressure wave. The sacculo-colic test could be used to assess otolith function in patients with balance symptoms.

\section{Audit-derived protocol for epistaxis management in north Glasgow: a study of 734 epistaxis admissions}

L Melia, O J Hilmi, G W McGarry

From the ENT Department, Glasgow Royal Infirmary, Glasgow, Scotland, UK

\section{Introduction}

Epistaxis is the commonest emergency in ENT in the UK. Best practice mandates direct therapy to shorten the in-patient episode. The literature suggests that hospital stay, transfusion requirement and operative intervention rates are surrogate measures useful in monitoring the quality of epistaxis management.

We aimed to audit epistaxis care within north Glasgow ENT services, with reference to key care indices.

\section{Methods}

The study took the form of a retrospective, structured audit. Key indices included length of in-patient episode, transfusion rate, treatment methods (i.e. packing, cautery or other), operative salvage rate, and timing and nature of salvage procedure (e.g. endoscopic spheno-palative artery ligation (ESPAL), internal maxillary artery ligation (IMAL), external carotid artery ligation (ECAL), septoplasty or other). A time line analysis examined the effects of treatment decisions on time to transfusion, time to surgery and admission duration.

We included patients presenting as emergency admissions with epistaxis to North Glasgow ENT services within a five year period (1999-2004).

\section{Results}

Seven hundred and thirty-four patients were admitted with epistaxis as the principal coded diagnosis (age range, 16-100 years; mean age, 67 years). The mean duration of admission was three days (minimum, one day; maximum, 35 days; mean, three days). Most patients were managed using indirect techniques (packing in 62 per cent), resulting in prolonged hospital stay. Transfusion was required in 16 of these cases (2.5 per cent). Seventy-two patients required surgery, 51 of whom had endured repeated packing before being taken to theatre (71 per cent) and 11 of whom required blood transfusion ( 15.3 per cent). The timing and distribution of these transfusions suggested that this decision was a pre-operative manoeuvre in patients destined for salvage surgery. Two of the theatre cases were readmitted with epistaxis ( 2.7 per cent), compared with 15 of the nontheatre cases (2.3 per cent). The average duration of hospital stay before surgery was four days; the average duration of hospital stay between surgery and discharge was one day. 


\section{Conclusions}

An unacceptably high proportion of patients were subjected to repeated, ineffective packing treatments - some patients were packed up to five times. Following surgical intervention, final discharge was achieved within 24 hours, with only a 2 per cent readmission rate.

We propose the following management protocol: direct therapy as the first-line treatment (with repeated packing forbidden) and early salvage surgery (endoscopic sphenopalative artery ligation (ESPAL)) advocated for all primary cases not controlled within 48 hours of admission. Early consultant involvement (within the first 24 hours) underpins this care strategy.

The effect of this policy will be the subject of a future, prospective audit.

\section{Evaluation of implementation of Scottish Intercollegiate} Guidelines Network 45 (antibiotic prophylaxis in surgery)

\section{M Wynne, L D Cooke}

From the ENT Department, Gartnavel General Hospital, Glasgow, Scotland, UK

Objective

We aimed to assess awareness and implementation of SIGN 45.

\section{Methods}

A postal questionnaire was sent to consultant and specialist registrars in ENT surgery within Scotland.

\section{Results}

Fifty-seven questionnaires were returned (58 per cent). Of those who answered, 43 per cent were aware of the SIGN 45 guidelines and 90 per cent felt that their current practice was within these guidelines.

For tonsillectomy, functional endoscopic sinus surgery, and clean head and neck surgery, 100 per cent of respondents stated that their practice was within the current guideline. Ninety per cent of respondents practising otological surgery stated their practice was within current recommendations. With regard to contaminated head and neck surgery, all practicing respondents administered antibiotic prophylaxis, which is the current recommendation. However, only 42 per cent of respondents gave the current recommended antibiotic combination. Due to ambiguity in the current guidelines, this would result in a cost ranging from $£ 6.38$ to $£ 177.66$ per patient. No respondent administered the recommended number of doses.

\section{Conclusion}

Awareness of the SIGN 45 guidelines must be increased. The difference between current antibiotic practice within head and neck surgery and the recommendation of the current guideline must be addressed when the guideline is reviewed; the guidelines must also be made more precise.

\section{Is direct access tonsillectomy viable?}

\section{H Jayasinghe, G M MacDougal}

From the Head and Neck Directorate, St Johns Hospital, Livingston, Scotland, UK

Adult patients requiring tonsillectomy within the Edinburgh region can now be referred directly by general practitioners (GPs) for pre-operative surgical assessment if they meet the Scottish Intercollegiate Guidelines Network guidelines. This study evaluated whether this direct access tonsillectomy service is a viable adjunct to ENT out-patient review.

A prospective review of 42 patients referred by direct referral between September and November 2005 was undertaken to assess whether they met the SIGN criteria for tonsillectomy. Fifteen of the GPs making referrals during that time were contacted to assess their satisfaction with the direct access service.

All 42 patients and 12 of the 15 GPs contacted responded to the study. Of the 42 patient referrals made via the direct access route, 41 (97.5 per cent) patients met the SIGN guidelines in full. Thirty-seven ( 88 per cent) had the referral pro forma completed in full to indicate that all the necessary criteria had been met for tonsillectomy. Sixty-six per cent of the GPs expressed satisfaction with the current direct access pathway and felt there had been an overall improvement in the referral process since the pathway had been implemented. Only 33 per cent of the GPs surveyed felt that ENT out-patient assessment was preferable prior to pre-operative assessment.

\section{Assessment of patient benefit following structural and functional external septorhinoplasty, using the Glasgow benefit inventory and visual analogue scores}

A Y Isa, N Balaji

From the Department of Otolaryngology and Head and Neck Surgery, Monklands Hospital, Airdrie, Scotland, UK

\section{Objective}

We aimed to subjectively assess the outcome in patients who had undergone external septorhinoplasty at Monklands Hospital between 1998 and 2004, using the Glasgow benefit inventory questionnaire and visual analogue scores.

\section{Materials and methods}

Patients were sent two questionnaires, and case notes were reviewed to elucidate clinical indication.

\section{Results}

Thirty-six patients responded, 24 men and 12 women, aged between 18 and 74 years. These patients' surgery had occurred 1.5 to 6.5 years previously. The Glasgow benefit inventory showed benefit in the total score (median 25) and the general sub-scale (median 29). Improvement in breathing (median eight) and in nasal shape (median eight) was reported, with good overall satisfaction (median nine). As expected, patients undergoing structural and functional septorhinoplasty reported a higher visual analogue score for breathing than did the structural group (median eight, versus 3.5, respectively). The Glasgow benefit inventory scores (total score and general sub-scale) and the visual analogue scores were significantly associated with each other between the two patient groups (i.e. structural versus structural and functional), except for the visual analogue score for breathing in the structural and functional patient group.

\section{Conclusions}

The Glasgow benefit inventory benefit is in keeping with the findings of other reports on septorhinoplasty. As a method of enabling more specific subjective assessment of nasal shape and breathing, the visual analogue scale is an easy, useful adjunct. 